\title{
Anisotropic elastic parameters for soil
}

PICKERING, D. J. (1970). Géotechnique 20, No. 3, 271-276. Discussion (1971) 21, No. 2, 181-183

\section{R. N. Chowdhury, Sheffield Polytechnic and G. J. W. King, University of Liverpool}

In reply to the Writers' (1971) discussion two examples have been considered by Pickering (1971) to determine the signs of the volumetric strain. In his example 1, the volumetric strain was of the same sign as the applied stresses $\sigma_{x}=\sigma_{y}=\sigma_{z}=\sigma$. However, he would certainly not claim this for all other possible states of stress. A condition on the values of the material parameters must embrace all possible stress states in the class for which it is derived. (It may for instance be derived for generalized stress for plane strain compression, etc.) Consider the restrictions derived by the Writers and the Author for incompressibility. The material parameters in example 1 of the Author do not obey these restrictions; yet there are many stress states for which the volumetric strain would be zero, e.g.

$$
\begin{aligned}
& \sigma_{x}=2 \sigma, \sigma_{y}=\sigma_{z}=\sigma \\
& \sigma_{x}=\sigma_{y}=\sigma, \sigma_{z}=2 \sigma \\
& \sigma_{x}=\sigma_{z}=1 \cdot 5 \sigma, \sigma_{y}=\sigma
\end{aligned}
$$

or any other values for which $3 \sigma_{y}-\sigma_{x}-\sigma_{i z}=0$.

Therefore, incompressibility may result for particular stress states with any values of the elastic parameters. This does not, however, prove that the restrictions on the values of the parameters are unnecessary, since the material could be subjected to other states of stress resulting in net volume change unless those restrictions were obeyed.

In deriving the conditions for the volumetric strain the tacit assumption made by the Writers was that all stress components are of the same sign; these conditions can not be derived otherwise. In many practical problems and widely used test systems (triaxial compression test and plane-strain compression test) the principal stresses are of the same sign but may assume a wide range of values. For such stress states, the derived conditions are necessary and sufficient for the volumetric strain to be the same sign as the applied stresses. The Writers' use of the term 'mean applied stress' perhaps explains why the Author chose principal stresses of unlike sign in his example 2. With a choice of like signs any numerical calculation will confirm the sufficiency of the derived conditions.

The practical importance of these conditions, namely $\mu_{y x}<0 \cdot 5, \mu_{x x}<1-\left(E_{x} / E_{y}\right) \mu_{y x}$ is best illustrated from studies of embankment stresses made by the Writers. When the assumed properties of the anisotropic elastic foundation of the embankment did not obey these conditions, the base of the embankment showed upward movement; when the conditions were obeyed, the movement was sensibly downwards as for an isotropic foundation. (Note that the principal stresses are compressive in most of the region directly beneath a built-up embankment.)

The Author agrees that the elastic parameters will need modification when soil is considered as a two-phase material and does not specifically disagree with the expression $\frac{1}{2}\{\bar{\sigma}\}^{T}[\bar{C}]\{\sigma\}$ for strain energy given by the Writers. This expression is not a quadratic form either of $\{\bar{\sigma}\}$ or of $\{\sigma\}$. Therefore the conditions that all the principal minors of $[\vec{C}]$ be positive for the strain energy to be positive (Hearmon, 1961) is not relevant and consequently it is doubtful whether the restrictions on the parameters constituting $[\bar{C}]$ are the same as on those consttuting $[\bar{C}]$. 
REFERENCE

Hearmon, R. F. S. (1961). An introduction to applied anisotropic elasticity. Oxford University Press.

\section{J. Pickering}

In reply to the first two paragraphs of this further correspondence, the Author thinks that the discussion is really an argument about the meaning of the word 'necessary'. Insofar as the sufficiency of the Writers' criteria are concerned, the Author agrees that if they add the condition that all normal stress components have the same sign, the criteria are sufficient to ensure that the volumetric strain and the mean stress have the same sign. However, this is fairly obvious, because one of their criteria is that quoted in the Paper, as the boundary of materials which dilate under compressive $\sigma_{y}$, and the other is the boundary of materials which dilate under compressive $\sigma_{x}$ or $\sigma_{z}$. Furthermore, although the limitation of the stress field in this way may be meaningful in the context of the Writers' particular application, it is a very rash assumption for general use. For example, consider the use of incremental stress fields or a case where pore pressure rises with one or some of the total stresses constant. The Author rather wonders about the value of these criteria. Material outside them may expand under mcan compression. It is known that such soils exist. The Paper showed that such transversely isotropic elastic materials can exist. The Writers may feel that it is realistic to have the base of their embankment moving down but it if is built on a dilatant material, parts of it may in fact move up. Why take such pains to decide criteria which are not necessary, are sufficient only if an arbitrary limitation on the stress field is included and which govern a condition which does not seem to have any very great significance?

Considering now the Writers' formulation $\frac{1}{2}\{\bar{\sigma}\}^{T}[\bar{C}]\{\sigma\}$, the Author agrees that this somewhat intractable expression represents the strain energy per unit volume for undrained soil. However, the elastic constants are limited by other considcrations, from which meaningful results can be obtained. In the Paper the Author developed the concept of a matrix of elastic constants $[C]$ for an idealized homogeneous material. Then, in considering a saturated soil, he applied $[C]$ explicitly to the soil skeleton. He thought he had made himself clear as to what he was doing and that he was tacitly assuming that the same bounds would govern $[C]$ when applied to the soil skeleton. He left out the justification of this assumption in the interests of brevity. In the next paragraphs the grounds of his assumption will be explained. The Author would have liked to continue to use $[C]$ for the soil skeleton and to put $[C]_{t}$ for the total stress parameters, acknowledging that they are a function of the degree of saturation and drainage and of assumptions regarding the compressibility of water, and so on, as well as of the soil skeleton. However, he realizes that it is more usual to use a notation such as $[\bar{C}]$ for the soil skeleton, relating strains to effective stresses, so he will adopt the notation used by the Writers.

The matrix $[\bar{C}]$ must be positive definite in order to ensure that the strain energy in a drained soil is positive. $[\vec{C}]$ will vary with conditions and assumptions, but for a given case, the total strain energy will be represented by $\frac{1}{2}\{\sigma\}^{T}[C]\{\sigma\}$ and $[C]$ must be positive definite. Thus, although the values of individual elements may be different, the limiting ratios of $[C]$ will bc the same as those required for $[\bar{C}]$ in drained conditions.

It remains to consider whether there are any further limitations to be imposed on $[\bar{C}]$ to ensure elastic stability under undrained conditions. The Author agrees that because $[\bar{C}]$ is positive definite, this fact alone does not ensure that $\frac{1}{2}\{\bar{\sigma}\}^{T}[\bar{C}]\{\sigma\}$ will be positive.

However, let it be accepted, in the interests of brevity, that the total strain energy is the sum of the strain energy of the soil skeleton and the pore fluid(s). Then if $[\bar{C}]$ is positive definite, the strain energy of the soil skeleton must be positive and, unless a pore fluid with negative 
compressibility can be found, the total strain energy of the two-phase system must be positive. The same limiting conditions therefore apply to $[\bar{C}]$ for undrained as for drained soil.

A pore fluid with negative compressibility is itself unstable and so ruled out of consideration in this context. However, if such a fluid were postulated, the Author agrees that it would place further restrictions on $[\bar{C}]$, which would depend on a sixth 'elastic' constant. Use of such a pore fluid in an undrained test would presumably make some soils explode!

In conclusion, the Author would like to remark that zero compressibility for pore water in undrained conditions is often assumed and this is represented by putting $\mu=0.5$ for total stress on isotropic soil. The corresponding manipulation for anisotropic soil of the type under discussion is to ensure that the values of $[\bar{C}]$ are such that the soil is represented by a point on line $\mathrm{AB}$ in Fig. 2 of the Paper. This does put a very severe limitation on the bounds of undrained $[C]$.

Finally the Author thanks the Writers for overlooking his absentminded use of the word 'idiomatic' when he meant 'axiomatic' in the previous correspondence.

\section{The dynamic penetration test: a standard that is not standardized}

IRELAND, H. O., MORETTO, O. and VARGAS, M. (1970). Géotechnique 20, No. 2, 185-192.

Discussion 20, No. 4, 452-456; (1971) 21, No. 2, 183-184; and No. 4, 423-424.

\section{S. K. Mathur, Lecturer in Civil Engineering, Institute of Technology, Banaras Hindu University, Varanasi-5 (India)}

The Paper is very informative because it describes the standard penetration test completely as used in North and South America, together with the modified and improved samplers and test procedures used in South America. The conflicting opinions expressed have doubtless created a lot of interest and enthusiasm. The Writer, however, shares the opinion expressed by the Authors that the S.P.T., having gained the confidence of a large number of professionals through its universal usage, should be standardized so that the results of the S.P.T. performed anywhere can be analyzed and compared without bothering about the variables connected with the type of apparatus used in each case.

The salient points of the I.S. Code 2131 (1963) pertaining to those raised in the Paper and in the Discussion by Drozd (1970) are given with a view to familiarizing everyone with the procedure adopted in India and are as follows.

(a) The borehole diameter can vary from 50 to $150 \mathrm{~mm}$.

(b) The drill rod should preferably have a stiffness equal to or greater that that of the Arod (41.3 mm o.d.). For depths of exploration greater than $10 \mathrm{~m}$, special precautions are required to keep the rod vertical by using spacers and/or by using stiffer rods to minimize the whipping effect.

(c) The driving head is of $65 \mathrm{~kg}$ weight with $75 \mathrm{~cm}$ free fall.

(d) Tests are made and samples taken at every change in stratum or at intervals not more than $1.5 \mathrm{~m}$, whichever is less.

(e) The number of blows required to effect each $15 \mathrm{~cm}$ of penetration is recorded. The first $15 \mathrm{~cm}$ of drive is considered to be a seating drive. The total blows required for the second and third $15 \mathrm{~cm}$ of penetration are termed the penetration resistance $N$. Refusal is considered to have been reached when the rate of advance is less than $2.5 \mathrm{~cm}$ for 50 blows. 